\title{
Production of recyclates - compared with virgin Plastics - a LCA Study
}

\author{
Birgit Kjaerside Storm* \\ Aalborg University Esbjerg, Niels Bohrs Vej 8, 6700 Esbjerg, Denmark \\ Plastix A/S, Gammel Landevej 3, 7620 Lemvig, Denmark
}

\begin{abstract}
Plastix A/S is a Danish cleantech company transforming discarded fishing trawls and nets into valuable green raw materials. Plastix' technology and processes solve a maritime waste problem and contribute to a more circular green economy and reduce landfilling, marine pollution, $\mathrm{CO}_{2}$ emissions and especially loss of valuable resources. Plastix' recycling technology enables recovery of discarded fishing trawls and nets via mechanical and thermal processes transforming the waste into valuable recycles which can be converted into plastic products replacing virgin raw materials. The performance has been proved through a Life Cycle Assessment (LCA) study. The results from the LCA study are compared with the production of virgin materials. The results of the LCA show that especially the carbon footprint is remarkable better for Oceanix than for virgin plastics. Oceanix HDPE is 5 times better than virgin HDPE, when talking about the carbon foot print, and the results for Oceanix PP and Oceanix PA6 are 5 times and 20 times better compared with virgin PP and PA6. Also other environmental indicators are better for Oceanix compared with virgin plastics.
\end{abstract}

\section{Introduction}

Plastix A/S is a Danish cleantech company transforming discarded fishing trawls and nets into valuable green raw materials. Plastix' technology and processes solve a maritime waste problem and contribute to a more circular green economy and reduce landfilling, marine pollution, $\mathrm{CO}_{2}$ emissions and especially loss of valuable resources. Plastix' recycling technology enables recovery of discarded fishing trawls and nets via mechanical and thermal processes transforming the waste into valuable recycles which can be converted into plastic products replacing virgin raw materials.

The materials collected and recycled in Plastix A/S are mainly high density polyethylene, HDPE, polypropylene, PP, and polyamide 6, PA6. They are the main part of the materials used for fishing trawls and nets used in the fishing industry. Plastix' cleantech recycling processes are as follows showing the four steps in the plant: 1. Gate control and storage; 2. Rough cleaning and cutting; 3. Cleaning and final separation; 4. Extrusion

After the extrusion all batched are controlled in the laboratory for measuring the main properties of the produced granulates.

* Corresponding author: bkstorm@bbsyd.dk 
The input materials - the fishing nets and trawls - are collected from different ports in Europe. Plastix A/S wants an input with as low part of other types of waste. For obtaining a good quality of fishing gears a point system for ports and other suppliers has been developed. They can get a certificate for the quality. The higher quality the better certificate the supplier will receive. In Figure 1 an example of the certificates is shown. The certificates can be that one shown in Figure 1, or it can be a bronze, silver or a golden certificate.

In the gate control the received materials are weighed and visually inspected for the quality. If there are too much other things in the container than fishing gears, the container can be sent back to the supplier or the prize for the materials can be reduced to that Plastix A/S shall have a prize for taking the material instead of paying something for the "raw" material. The container receive a number, so it after it possible during the production to find back to input material.

The raw materials are stored in the grey zone. The grey zone is the place, where the input materials are received and roughly sorted up. The grey zone is placed outdoors. In the grey zone the net and trawls are sorted in different materials. Other parts - as for example ropes and floaters - are cut off

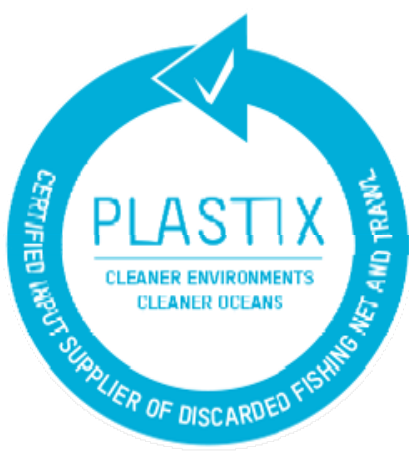

Fig. 1. An example of a certificate for a supplier to Plastix A/S. and stored for being either sold or also recycled. Totally Plastix A/S has a very small amount of waste, because all types of materials are regarded as a resource.

From the grey zone the materials are sent to the green zone, where it will be cut into pieces so most of the knots are cut and the materials are in pieces, so a washing of it can be made. Quite a big part of the sand in the fishing gears are removed in the grey zone as well. In the green zone it is a problem for the cutting equipment that there is sand left in the fishing gear, because it causes wear on the knives. The polyamide is a quite tough material, and it itself is a challenge to cut. That combined with the sand gives wear on the knives. Plastix A/S has its own mechanical workshop to develop and to maintain the machinery.

From the grey zone the materials are transferred to the blue zone, where it is washed

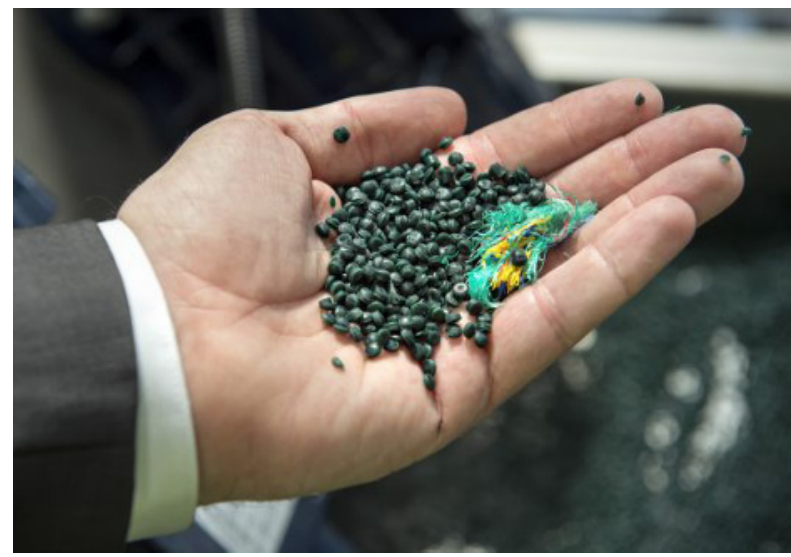

Fig. 2. Raw materials of Polyethylene and granulates of polyethylene. (Photo made at Plastix A/S) and sorted out in different fractions, so only a minor part of polyamide will go into the polyethylene fraction, and a minor part of polyethylene will go into the polyamide fraction. A minor part of PP in HDPE and HDPE in PP can be accepted, because those materials are compatible if only a small amount of contamination part is present. In the blue zone the washed materials are dried, too, so dry materials are sent to the white zone, which is the extrusion zone. Here the cut, washed and dried materials will be made to granulate or pellets 
and stored in big bags before being delivered to the customer. In Figure 2 granulates can be seen together with a piece of the raw materials. The granules and the raw materials shown in the figure are polyethylene.

A laboratory control of all inputs and outputs is made. The input is control for the type of polymer. During the production the content of sand and water in the washed and dried material are measured before sending it to the extruder.

The output is controlled for strength, flexibility, density and melt flow index. All those results are used for the technical datasheets for the recycles.

Plastix A/S has decided to call the recyclates made from fishing gears for Oceanix, which then become Oceanix PA, Oceanix HDPE and Oceanix PP.

For improving that it is a clean tech product Plastix A/S makes a life cycle assessment has been made for the products.

\section{Life Cycle Assessment}

Life Cycle Assessment, LCA in the following, is a tool for systematic evaluation of the environmental aspects of a product or service system through all stages of its life cycle. LCA provides an adequate instrument for environmental decision support. Reliable LCA performance is crucial to achieve a life cycle economy. The International Organization for Standardization (ISO) has standardized this framework within the series ISO 14040 on LCA.

For making a Life Cycle Assessment ISO 14040 and ISO 14044 is used. A LCA is often called a "cradle-to-grave" or a "cradle-to-cradle" analysis depending on, how the analysis is made. A "cradle-to-gate" analysis can be made as well. In this case the analysis is called a "grave-to-cradle" analysis, because Plastix A/S use discarded materials as raw materials and convert it to a new raw material.

For evaluation of the LCA a functional unit needs to be decided for comparison of the results as well as system boundaries need to be defined. The stages in LCA are:
1. Raw material stage
2. Manufacturing stage
3. Use stage
4. Waste stages - or end of use stage
5. Transport stage.

For all stages the same functional unit needs to be regarded. The system boundaries are set up, so they will give the true picture - or a picture as true as possible - of the product regarded $[1,2]$.

\section{Life Cycle Assessment for Oceanix recyclates}

All the calculations in the LCA for Oceanix have been performed by Provice by Tomas Sander Poulsen [3]. The LCA has been performed as a part of a project co-funded by Ecoinnovation Initiative of the European Union, Retrawl.

According to the standards mentioned above the functional unit in the study is defined as 1 ton of produced plastic granulates output of the three materials PA6, HDPE and PP.

The LCA calculations in the report are conducted by Simapro calculation tool and the Ecoinvent database $[4,5]$.

By defining the unit as stated above, a closed loop assessment of discarded trawl and net produced from recycled or virgin plastic can also be conducted.

In this case a comparison study of discarded materials and virgin materials are compared. For making that possible the used model is a "cradle-to-gate" analysis is 
performed for both type of materials, even that the right analysis for the recycles should be a "grave-to-cradle" analysis, but that would give a wrong picture for comparison with virgin materials.

The trawl and net can be delivered from different harbors situated $20 \mathrm{~km}$ away or 1500 $\mathrm{km}$ away from Plastix A/S manufacturing plant. In the report a distance for the transport and the transport system has been $1250 \mathrm{~km}$, where $1000 \mathrm{~km}$ are covered with ship and 250 $\mathrm{km}$ with truck. In Figure 3 the system boundaries for the data collection are shown.

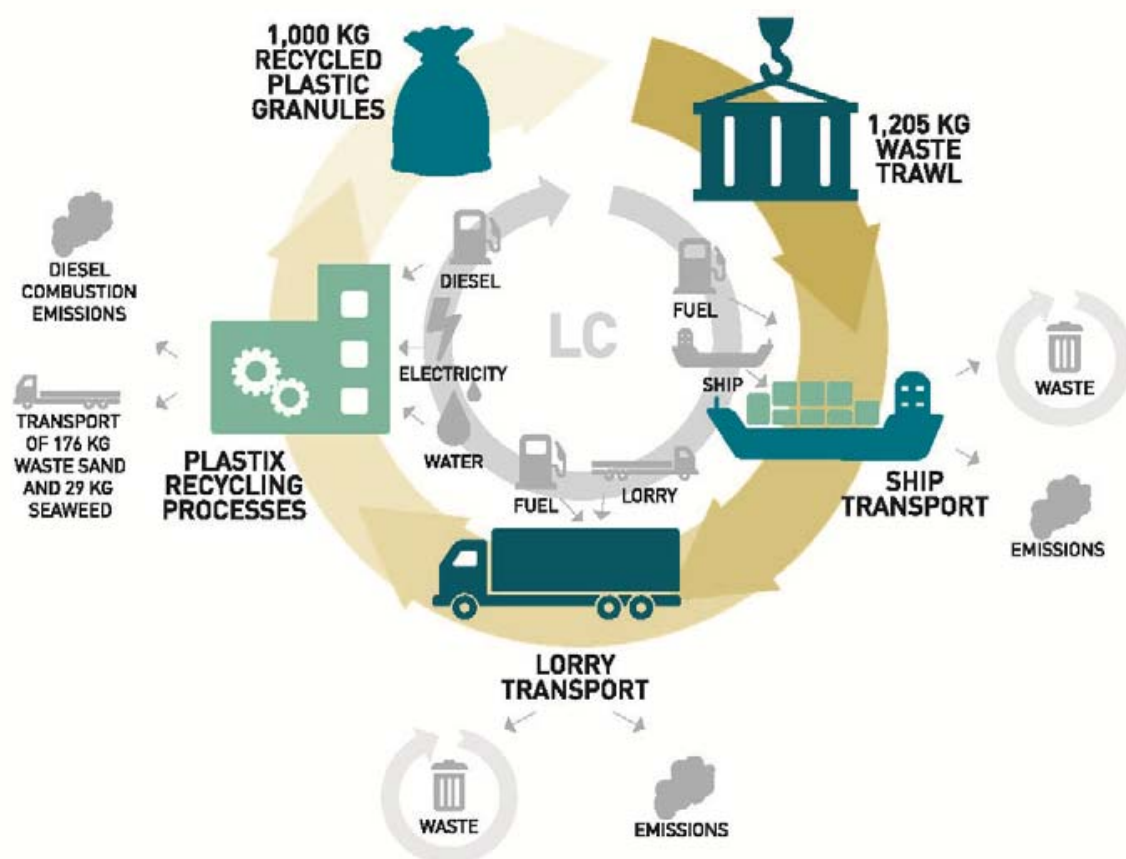

Fig. 3. System boundary of data collection for Plastix recycling scenario including the mass balance [3].

As illustrated in the above shown Figure 3, the process flow of the recycling scenario of PA6, PE and PP has a life cycle consisting of the following steps:

1. Transport of discarded waste to Plastix A/S manufacturing plant

2. The recycling processes at Plastix A/S to final recycles.

Plastix' cleantech recycling processes are as follows showing the four steps in the plant:

1. Gate control and storage

2. Rough cleaning and cutting

3. Cleaning and final separation

4. Extrusion

In the LCA it has been used that 1 ton of recycled plastic granulates will need $1,075 \mathrm{~kg}$ discarded net and trawl, because it is assumed that there will be sand and organic matter corresponding to approximately $75 \mathrm{~kg}$ per 1000 plastic. This number is set as an average part, because as mentioned above there are different amounts of sand and other materials in the received raw material.

The processes for cleaning, cutting, washing, separation, drying and extrusion use energy. The amount of energy used per ton plastic depends on the sort of plastic. Internal transport is also an energy consuming process and is calculated as well. In Figure 3 all 
different contribution to energy consumption are listed together with the emissions to the environment and waste from the production.

In the production washing water is cleaned and recirculated. Those processes are calculated in the LCA.

All data related to the Life Cycle Inventory of the virgin plastic production are collected from the Ecoinvent database [5], and no site specific data have been added. Figure 4 shows the system boundaries of data collection and process flow for the virgin product scenario.

The LCA for the virgin products use non-renewable resources as naphtha and benzene for PE and PP and for PA6, caprolactame made from crude oil is used as raw material. The product system for making virgin plastic consists of the sub-processes:

1. Mining/production of raw materials

2. Refining of raw materials (producing monomers and other raw materials)

3. Additional processing

4. Polymerization

5. Transport

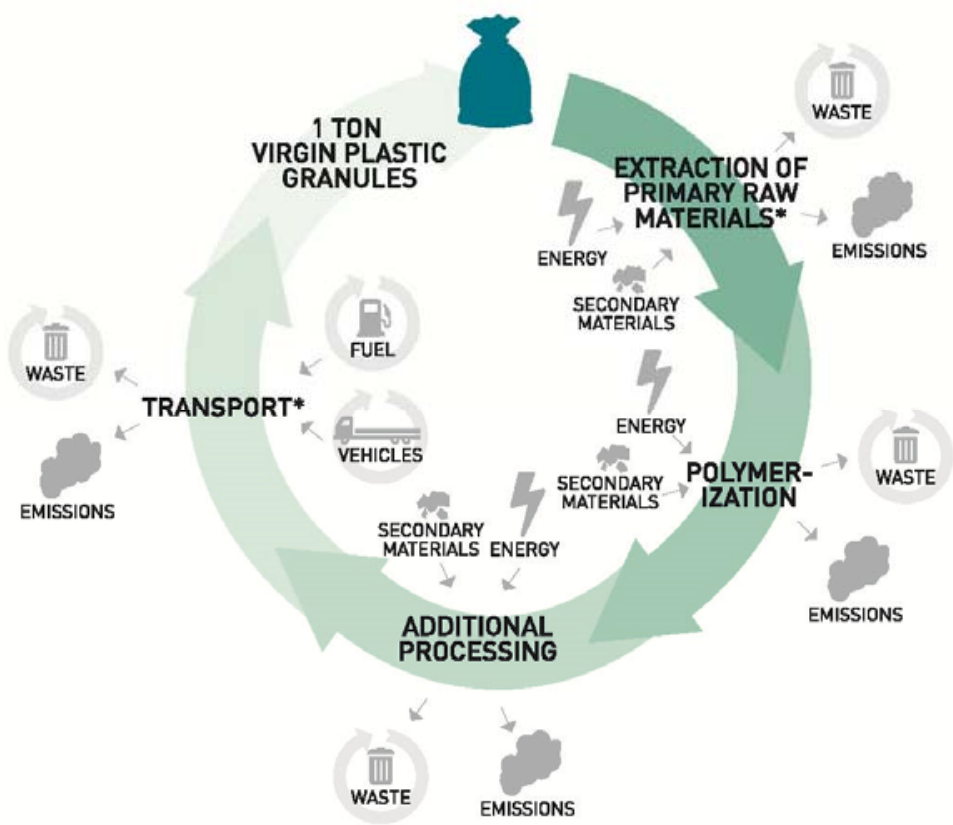

Fig. 4. System boundaries of the data collection and process flow for the virgin plastic production scenario [3].

The LCA calculations in the report are conducted by Simapro [4] calculation tool and the Ecoinvent database [5].

The impact categories included in the LCA are a shortlist of environmental impacts calculated in SimaPro. The impact categories are: Climate Change, Terrestrial acidification, Marine and fresh water eutrophication, photochemical oxidant formation, Particulate matter formation, Marine eco toxicity, Fossil depletion.

Climate change is mainly calculated as the contribution of $\mathrm{CO}_{2}$ from the processes and is illustrated by the carbon foot prints in the processes. The $\mathrm{CO}_{2}$ is calculated because it has a global warming potential, GWP. For $\mathrm{CO}_{2}$ the potential is set to 1 . Other matters have a higher GWP, but none of them are significant here.

Each single process emits different substances and they may or may not contribute to a category. 
The environmental impacts of the processes at Plastix $\mathrm{A} / \mathrm{S}$ are mainly related to electricity consumption, water consumption, landfill avoidance and transport activities. The results show that the recycling processes at Plastix A/S outperforms the virgin plastic scenario. The main part of the contributions is from the electricity consumption at Plastix A/S, more specifically the part of non-renewable coal energy sources $(23 \%)$ that supply the electricity production. The electricity consumption of the recycling process is small compared to the energy consumption from virgin plastic processes. Even with these impacts from electricity use, the Plastix A/S process outperforms the virgin plastic scenario. If the electricity used by Plastix A/S came from renewable energy sources, the impacts from the recycling process would be close to none, but until now that's not the case.

Energy use is the largest input in the Plastix A/S recycling process. A comparison only on energy can be made, since PlasticsEurope [6-8] reports inform of the energy required to make the different virgin plastics.

Table 1 the different energy consumption levels are illustrated.

Table 1. Energy consumption per ton produced virgin plastic and Plastix plastic (Oceanix). Plastix total energy consumption is only $5-11 \%$ of virgin plastic energy consumption [3].

\begin{tabular}{|l|l|l|l|l|l|l|}
\hline & $\begin{array}{l}\text { Virgin } \\
\text { PA }\end{array}$ & $\begin{array}{l}\text { Plastix } \\
\text { PA }\end{array}$ & $\begin{array}{l}\text { Virgin } \\
\text { PE }\end{array}$ & $\begin{array}{l}\text { Plastix } \\
\text { PE }\end{array}$ & $\begin{array}{l}\text { Virgin } \\
\text { PP }\end{array}$ & $\begin{array}{l}\text { Plastix } \\
\text { PP }\end{array}$ \\
\hline $\begin{array}{l}\text { Process energy } \\
\text { Consumption } \\
\text { per ton (kWh) }\end{array}$ & 25,167 & 1,257 & 9,444 & 922 & 8,750 & 976 \\
\hline $\begin{array}{l}\text { Plastix energy } \\
\text { consumption } \\
\text { in relation to } \\
\text { virgin energy } \\
\text { consumption } \\
\text { \% }\end{array}$ & $5,0 \%$ & & $9,8 \%$ & & $11,2 \%$ \\
\hline
\end{tabular}

All the results of the calculations of the environmental impacts for as well Plastix A/S materials (Oceanix) as for the virgin plastics are collected in Figure 5. All results are normalized to a European citizen's person equivalent. Figure 5 determines that the Plastix $\mathrm{A} / \mathrm{S}$ impacts are significantly lower than all the virgin plastic scenario in all the environmental categories except fresh water eutrophication and marine eco toxicity. Both impacts are related to indirect emissions from consumption of non-renewable coal-based energy.

The results of the LCA for Plastix A/S recycled plastics (Oceanix) and the comparison between the virgin plastics are shown in Figure 5.

Even when the results have been calculated for a worst case scenario for Plastix' products, the LCA shows better results than for virgin materials. The worst case scenario has been calculated for

- amount of input sand transported with waste trawl is $15 \%$ instead of $5 \%$, handled in process and disposed toll landfill;

- transport scenario is $650 \mathrm{~km}$ by truck and $600 \mathrm{~km}$ by ship instead of $250 \mathrm{~km}$ by truck and $1000 \mathrm{~km}$ by ship.

The worst case scenario shows still reduction in the carbon footprint for being 17 times better for the recycled PA6 than for virgin PA6, and 4 times better for recycled PE and PP compared with virgin $\mathrm{PE}$ and $\mathrm{PP}$. 


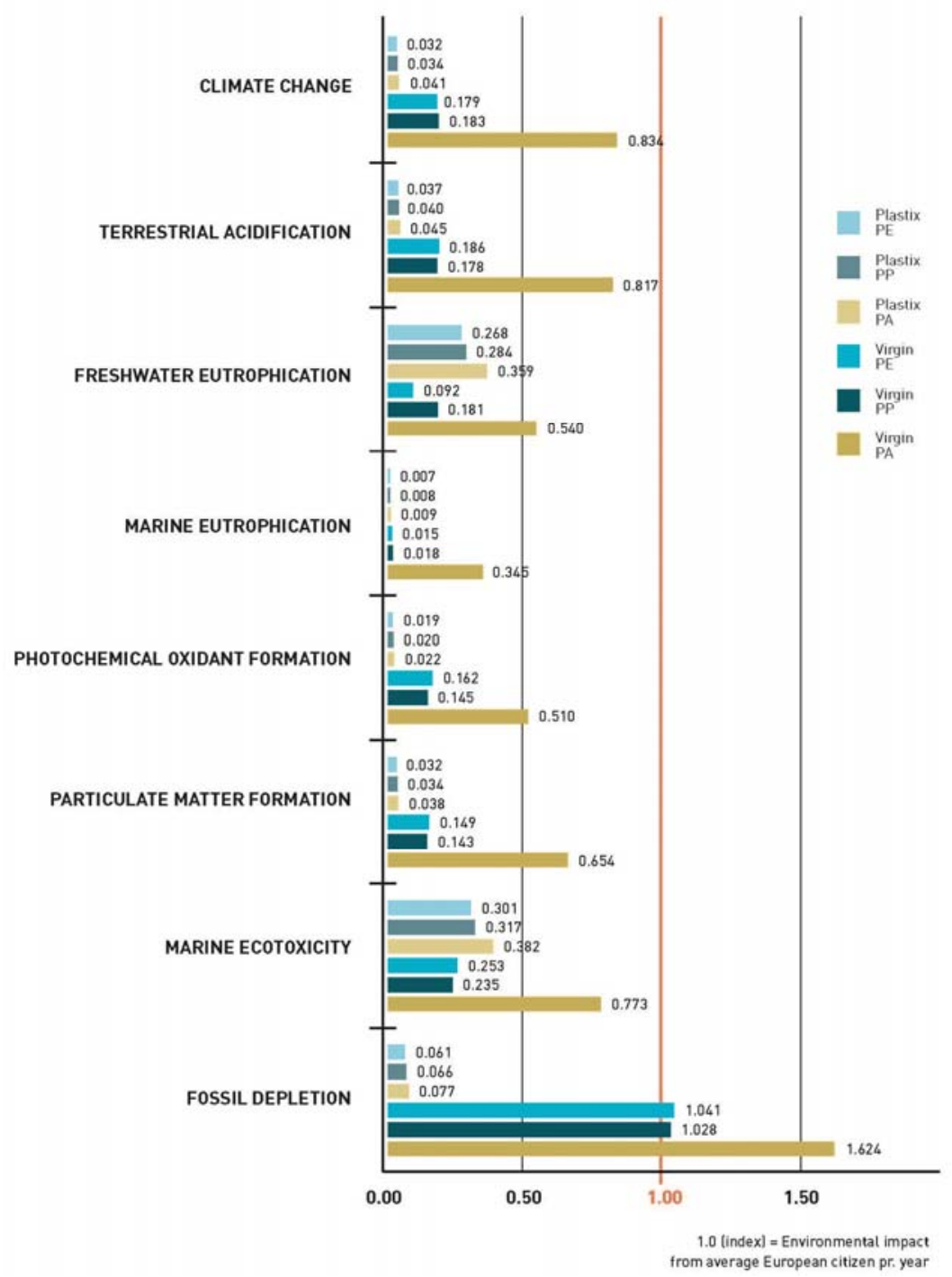

Fig. 5. LCA results of environmental impacts from one tones produced plastics with Plastix recycling technology and virgin plastic production (normalized to a European citizens person equivalent) [3].

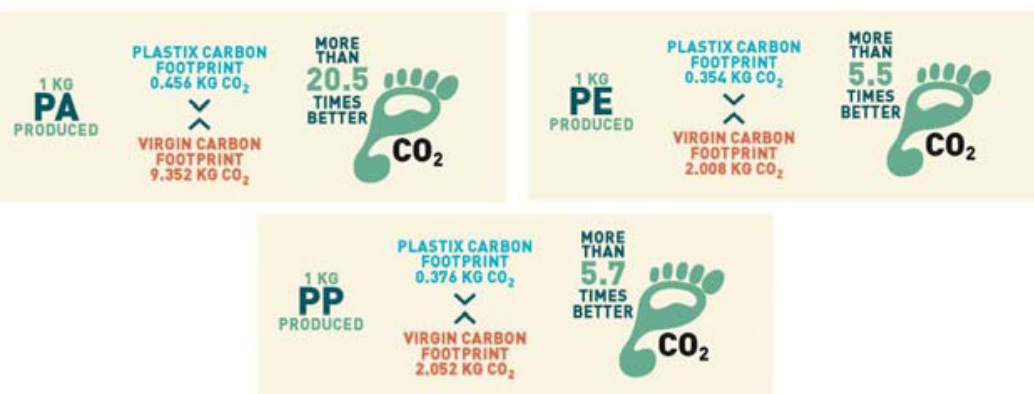

Fig. 6. Carbon footprint for Plastix A/S recycled plastic, Oceanix, compared with virgin plastics [4]. 
The reduction in the carbon footprint, which has been regarded as being important for climate change considerations are as shown in Figure 6. The reduction in carbon footprint for PA6 is 20 times compared with virgin PA6, and 5 times for PE and PP compared with virgin $\mathrm{PE}$ and $\mathrm{PP}$.

\section{Conclusion}

Plastix A/S use discarded fishing nets and trawls transforming tem to new raw materials for use in the plastic industry. The discarded nets and trawls are treated through a separation process, a cutting process, a washing process and an extrusion process to granulate called Oceanix. The polymers in the nets and trawls are HDPE and PA6 and in ropes PP.

The LCA results illustrates that the environmental impacts and here especially the carbon footprint also regarded as climate change of Plastix' cleantech technology and processes for recycling of discarded trawls and nets are substantially better than the production of virgin plastic of the same types.

With regard to fossil depletion the Plastix A/S recycling scenario performs by a factor 17 better than virgin PE, a factor 16 better than virgin PP and a factor 21 better than virgin PA.

With regard to the carbon footprint the recycling scenario performs by a factor 5.7 for PE, 5.5 for PP and 20.5 for PA compared with virgin PE, PP and PA.

\section{References}

1. ISO 14040, LCA principles and framework (2016)

2. ISO 14044, LCA requirements and guidelines (2016)

3. Plastix and Provice; Life Cycle Assessment of The Retrawl Project (2016)

4. Simapro LCA calculation tool version 8.2

5. Ecoinvent LCA database version 3.2

6. Plastic Europe: Ecoprofiles and Environmental Product Declarations Report, on PA6 (2014)

7. Plastic Europe: Ecoprofiles and Environmental Product Declarations Report, on PE (2014)

8. Plastic Europe: Ecoprofiles and Environmental Product Declarations Report, on PP (2014) 